\title{
LV-PP-4-5
}

\section{Laparoscopic and robotic volume preserving major hepatectomy}

\author{
Jin Hong LIM, Dong Sup YOON*
}

Division of Hepatobiliary Pancreatic Surgery, Department of Surgery, Gangnam Severance Hospital, Yonsei University College of Medicine, Seoul, Korea

Introduction: Transection along the right anterior fissure was proposed as a mechanism by which to open the third door of the liver for anatomical hepatectomy. Nowadays, laparoscopic anatomical liver resection is gaining momentum with less blood loss and shorter postoperative hospital stay. In this study, we sought to evaluate the feasibility, safety and potential benefits of laparoscopic assisted volume preserving major hepatectomy.

Methods: Between October 2016 and December 2020, 76 patients underwent volume preserving major hepatectomy at the authors' institution. Except 3 patients underwent combined CBD resection, 45 patients underwent laparoscopic (42 patients) or robotic (3 patients) volume preserving major hepatectomy and 31 patients underwent open volume preserving major hepatectomy. Patient demographics and perioperative outcomes were analyzed and compared.

Results: There was no open conversion during laparoscopic procedure. There were no differences in operation time and length of hospital stay between laparoscopic assisted and open ventral segment preserving right hepatectomy. Patients who underwent laparoscopic procedure had less bleeding amount than those who underwent open procedure.

Conclusions: Laparoscopic and robot volume preserving major hepatectomy is a feasible and safe procedure. However, more experience is essential to reveal the long term oncological results of volume preserving major hepatectomy. 